\title{
New gaps between zeros of fourth-order differential equations via Opial inequalities
}

\author{
SH Saker $^{1 *}$, RP Agarwal ${ }^{2}$ and D O'Regan ${ }^{3}$
}

${ }^{\text {"Correspondence: }}$

shsaker@mans.edu.eg

'Department of Mathematics,

Faculty of Science, Mansoura

University, Mansoura, 35516, Egypt

Full list of author information is

available at the end of the article

\begin{abstract}
In this paper, for a fourth-order differential equation, we will establish some lower bounds for the distance between zeros of a nontrivial solution and also lower bounds for the distance between zeros of a solution and/or its derivatives. We also give some new results related to some boundary value problems in the theory of bending of beams. The main results will be proved by making use of some generalizations of Opial and Wirtinger-type inequalities. Some examples are considered to illustrate the main results.
\end{abstract}

MSC: $34 \mathrm{~K} 11 ; 34 \mathrm{C} 10$

Keywords: Opial and Wirtinger inequalities; fourth-order differential equations; bending of beams

\section{Introduction}

In this paper, we are concerned with the lower bounds of the distance between zeros of a nontrivial solution and also lower bounds of the distance between zeros of a solution and/or its derivatives for the fourth-order differential equation

$$
\left(r(t) x^{\prime \prime \prime}(t)\right)^{\prime}+q(t) x(t)=0, \quad t \in \mathbb{I},
$$

where $r, q: \mathbb{I} \rightarrow \mathbb{R}$ are continuous measurable functions and $\mathbb{I}$ is a nontrivial interval of reals. By a solution of (1.1) on the interval $J \subseteq \mathbb{I}$, we mean a nontrivial real-valued function $x \in C^{3}(J)$, which has the property that $r(t) x^{\prime \prime \prime}(t) \in C^{1}(J)$ and satisfies equation (1.1) on $J$. We assume that (1.1) possesses such a nontrivial solution on $\mathbb{I}$. An existence result in the literature, which is related to our results in this paper, was proved by Lyapunov [1] for second-order differential equations. This result states that if $x(t)$ is a solution of the differential equation

$$
x^{\prime \prime}(t)+q(t) x(t)=0, \quad t \in \mathbb{R},
$$

with $x(\alpha)=x(\beta)=0(\alpha<\beta)$ and $x(t) \neq 0$, then

$$
\int_{\alpha}^{\beta}|q(t)| d t>\frac{4}{(\beta-\alpha)}
$$

where $q$ is a real valued continuous function. This was strengthened in [2] with $|q(t)|$ replaced by $q^{+}$where $q^{+}=\max \{q(t), 0\}$. Since the appearance of this inequality various proofs

\section{Springer}

(c) 2012 Saker et al.; licensee Springer. This is an Open Access article distributed under the terms of the Creative Commons Attribution License (http://creativecommons.org/licenses/by/2.0), which permits unrestricted use, distribution, and reproduction in any medium, provided the original work is properly cited. 
and generalizations or improvements have appeared in the literature for different types of differential equations. For contributions, we refer the reader to the papers [3-22] and the references cited therein.

The nontrivial solution $x(t)$ of (1.1) is said to oscillate or to be oscillatory, if it has arbitrarily large zeros. Equation (1.1) is oscillatory if one of its nontrivial solutions is oscillatory. Equation (1.1) is said to be $(i, j)$-disconjugate if $i$ and $j$ are positive integers such that $i+j=4$ and no solution of (1.1) has an $(i, j)$-distribution of zeros, i.e., no nontrivial solution has a pair of zeros of multiplicities $i$ and $j$, respectively. In general, an $n$th order differential equation

$$
x^{(n)}(t)+q(t) x(t)=0,
$$

is said to be $(k, n-k)$-disconjugate on an interval $I$ in case no nontrivial solution has a zero of order $k$ followed by a zero of order $n-k$. This means that, for every pair of points $a, b \in I, a<b$, there does not exist a nontrivial solution of (1.1) which satisfies

$$
\left.\begin{array}{l}
x^{(i)}(a)=0, \quad i=0, \ldots, k-1, \\
x^{(j)}(b)=0, \quad j=0, \ldots, n-k-1 .
\end{array}\right\}
$$

The least value of $b$ such that there exists a nontrivial solution which satisfies (1.5) is called the $(k, n-k)$-conjugate point of $a$. The differential equation (1.4) is said to disconjugate on an interval $I$ if one of its nontrivial solutions has at most $n-1$ zeros. For our case, if no nontrivial solution of (1.1) has more than three zeros, the equation is termed disconjugate. Together with $(k, n-k)$-disconjugacy, we consider the related concept which is $(k, n-k)$ disfocality. The differential equation (1.4) is said to be disfocal on an interval $I$ if, for every nontrivial solution $x$ at least one of the functions $x, x^{\prime}, \ldots, x^{(n-1)}$ does not vanish on $I$. If the equation is not disfocal on $I$, then there exists an integer $k(1 \leq k \leq n-1)$, a pair of points $a, b \in I, a<b$ and a nontrivial solution $x$ such that $k$ of the functions $x, x^{\prime}, \ldots, x^{(n-1)}$ vanishes at $a$ and the remaining $n-k$ functions at $b$, i.e.,

$$
\left.\begin{array}{l}
x^{(i)}(a)=0, \quad i=0, \ldots, k-1, \\
x^{(j)}(b)=0, \quad j=k, \ldots, n-1 .
\end{array}\right\}
$$

Equation (1.1) is said to be (2,2)-disconjugate on $[a, b]$ if there is no nontrivial solution $x(t)$ and $c, d \in[a, b], c<d$ such that $x(c)=x^{\prime}(c)=x(d)=x^{\prime}(d)=0$. Equation (1.1) is said to be $(k, 4-k)$-disfocal on an interval $I$ for some $1 \leq k \leq 3$ in case there does not exist a solution $x$ with a zero of order $k$ followed by a zero of $x_{*}^{(j)}$ of order $4-k$, where $x_{*}^{(j)}=x^{(j)}$ for $j=0,1,2,3$ and $x_{*}^{(4)}=\left(r x^{\prime \prime \prime}\right)^{\prime}$. For $n$th order differential equations $(k, n-k)$-disconjugacy and disfocality are connected by the result of Nehari [23] which states that if (1.4) is $(k, n-k)$-disfocal on $(a, b)$ it is disconjuguate on $(a, b)$. For more details about disconjugacy and disfocality and the relation between them, we refer the reader to the paper [24].

In [4] and [25], the authors established some new Lyapunov type inequalities for higher order differential equations. Next, we present some special cases of their results for fourthorder differential equations. In [25], it is proved that if $x(t)$ is a solution of the fourth-order differential equation

$$
x^{(4)}(t)+q(t) x(t)=0,
$$


which satisfies $x(a)=x^{\prime}(a)=x(b)=x^{\prime}(b)=0$, then

$$
\int_{a}^{b}|q(t)| d t \geq \frac{192}{(b-a)^{3}}
$$

and if $x(t)$ satisfies $x(a)=x^{\prime \prime}(a)=x(b)=x^{\prime \prime}(b)=0$, then

$$
\int_{a}^{b}|q(t)| d t \geq \frac{4}{(b-a)^{2}}
$$

In [4], the author proved that if $x(t)$ is a solution of (1.7), which satisfies $x(a)=x(b)=x^{\prime \prime}(a)=$ $x^{\prime \prime}(b)=0$, then

$$
\int_{a}^{b}|q(t)| d t \geq \frac{16}{(b-a)^{3}}
$$

In this paper, we are concerned with the following problems for the general equation (1.1):

(i) obtain lower bounds for the spacing $\beta-\alpha$, where $x$ is a solution of (1.1) satisfying $x^{(i)}(\alpha)=0$ for $i=0,1,2$ and $x^{\prime \prime}(\beta)=0$,

(ii) obtain lower bounds for the spacing $\beta-\alpha$, where $x$ is a solution of (1.1) satisfying $x^{(i)}(\beta)=0$ for $i=0,1,2$ and $x^{\prime \prime}(\alpha)=0$.

We will also establish some new results related to some boundary value problems in the theory of bending of beams. In particular, we consider the boundary conditions

$$
x(\alpha)=x^{\prime}(\alpha)=x(\beta)=x^{\prime}(\beta)=0,
$$

which correspond to a beam clamped at each end. Second, we consider the boundary conditions

$$
x(\alpha)=x^{\prime}(\alpha)=x^{\prime \prime}(\beta)=x^{\prime \prime \prime}(\beta)=0,
$$

which correspond to a beam clamped at $t=\alpha$ and free at $t=\beta$. The study of the boundary conditions $x(\beta)=x^{\prime}(\beta)=x^{\prime \prime}(\alpha)=x^{\prime \prime \prime}(\alpha)=0$ which correspond to a beam clamped at $t=\beta$ and free at $t=\alpha$, and the boundary conditions $x(\alpha)=x^{\prime \prime}(\alpha)=x(\beta)=x^{\prime \prime}(\beta)=0$, which correspond to a beam hinged or supported at both ends are similar to the proof of the boundary conditions (1.8)-(1.9) and will be left to the interested reader. For more discussions of boundary conditions of the bending of beams, we refer to [26, 27].

The paper is organized as follows: In Section 2, we prove several results related to the problems (i)-(ii) and also prove some results related to the boundary value problems of the bending of beams with the boundary conditions (1.8) and (1.9). The main results will be proved by employing some Opial and Wirtinger type inequalities. The results yield conditions for disfocality and disconjugacy. In Section 3, we will discuss some special cases of the results to derive some new results for equation (1.7) and give some illustrative examples. To the best of the authors knowledge, this technique has not been employed before on equation (1.1), and the ideas are different from the techniques employed in [4] and [25]. We note of particular interest in this paper is when $q$ is oscillatory. 


\section{Main results}

In this section, we will prove the main results by employing some Opial and Wirtinger type inequalities. In the following, we present a generalization of Opial's inequality due to Agarwal and Pang [28, Theorem 3.9.1] that we will need in the proof of the main results.

Theorem 2.1 [28, Theorem 3.9.1] Assume that the functions $\vartheta$ and $\phi$ are non-negative and measurable on the interval $(\alpha, \beta), m, n$ are real numbers such that $\mu / m>1$, and $0 \leq$ $k \leq n-1(n \geq 1)$ fixed. Let $x \in C^{(n-1)}[\alpha, \beta]$ be such that $x^{(n-1)}(t)$ is absolutely continuous on $(\alpha, \beta)$. If $x^{(i)}(\alpha)=0$ for $k \leq i \leq n-1(n \geq 1)$ then

$$
\int_{\alpha}^{\beta} \phi(t)\left|x^{(k)}(t)\right|^{l}\left|x^{(n)}(t)\right|^{m} d t \leq K_{1}(\alpha, \beta)\left[\int_{\alpha}^{\beta} \vartheta(t)\left|x^{(n)}(t)\right|^{\mu} d t\right]^{(l+m) / \mu},
$$

where

$$
\begin{aligned}
& K_{1}(\alpha, \beta):=\frac{\left(\frac{m}{l+m}\right)^{\frac{m}{\mu}}}{((n-k-1) !)^{l}}\left[\int_{\alpha}^{\beta}\left(\phi^{\mu}(t) \vartheta(s)^{-m}(t)\right)^{1 /(\mu-m)}\left(P_{1, k}(t)\right)^{l(\mu-1) /(\mu-m)} d t\right]^{\frac{\mu-m}{\mu}}, \\
& P_{1, k}(t):=\int_{\alpha}^{t}(t-s)^{(n-k-1) \mu /(\mu-1)}(\vartheta(s))^{-1 /(\mu-1)} d s .
\end{aligned}
$$

If we replace $x^{(i)}(\alpha)=0$ by $x^{(i)}(\beta)=0, k \leq i \leq n-1(n \geq 1)$, then (2.1) holds where $K_{1}$ is replaced by $K_{2}$ which is given by

$$
K_{2}(\alpha, \beta):=\frac{\left(\frac{m}{l+m}\right)^{\frac{m}{\mu}}}{((n-k-1) !)^{l}}\left[\int_{\alpha}^{\beta}\left(\phi^{\mu}(t) \vartheta^{-m}(t)\right)^{1 /(\mu-m)}\left(P_{2, k}(t)\right)^{l(\mu-1) /(\mu-m)} d t\right]^{\frac{\mu-m}{\mu}},
$$

where

$$
P_{2, k}(t):=\int_{t}^{\beta}(s-t)^{(n-k-1) \mu /(\mu-1)}(\vartheta(s))^{-1 /(\mu-1)} d s .
$$

In the following, we present a special case of the Wirtinger type inequality due to Agarwal et al. [29] that we will need in the proof of the main results. For $\mathbb{I}=[\alpha, \beta]$ and a positive function $\lambda \in C^{1}(\mathbb{I})$ with either $\lambda^{\prime}(t)>0$ or $\lambda^{\prime}(t)<0$ on $\mathbb{I}$, we have

$$
\int_{\alpha}^{\beta} \frac{\lambda^{2}(t)}{\left|\lambda^{\prime}(t)\right|}\left|y^{\prime}(t)\right|^{2} d t \geq \frac{1}{4} \int_{\alpha}^{\beta}\left|\lambda^{\prime}(t)\right||y(t)|^{2} d t
$$

for any $y \in C^{1}(\mathbb{I})$ with $y(\alpha)=0=y(\beta)$.

Remark 1 It is clear that the inequality (2.4) is satisfied for any function $y$ satisfying the imposed assumptions. If we put $y(t)=x^{\prime \prime}(t)$ with $x^{\prime \prime}(\alpha)=0=\lambda(\beta)$ or $x^{\prime \prime}(\beta)=0=\lambda(\alpha)$, or $x^{\prime \prime}(\alpha)=0=x^{\prime \prime}(\beta)$ and $Q(t)=\lambda^{\prime}(t)$, then we have the following inequality which gives a relation between $x^{\prime \prime}(t)$ and $x^{\prime \prime \prime}(t)$ on the interval $[\alpha, \beta]$.

Lemma 2.1 For $\mathbb{I}=[\alpha, \beta]$, then we have

$$
\int_{\alpha}^{\beta}|r(t)|\left|x^{\prime \prime \prime}(t)\right|^{2} d t \geq \frac{1}{4} \int_{\alpha}^{\beta}|Q(t)|\left|x^{\prime \prime}(t)\right|^{2} d t
$$


for any $x \in C^{3}(\mathbb{I})$ with $x^{\prime \prime}(\alpha)=0=x^{\prime \prime}(\beta)$, where $r(t)$ satisfies the equation

$$
\left(r(t)\left(\lambda^{\prime}(t)\right)\right)^{\prime}-2 Q(t) \lambda(t)=0
$$

for any function $\lambda(t)$ satisfies $\lambda^{\prime}(t) \neq 0$.

Remark 2 Note that equation (2.6) holds if one chooses $r(t)=Q(t)=1$, where in this case

$$
\lambda(t)=\exp \sqrt{2} t
$$

Also, the inequality (2.5) holds if $r(t)=Q(t)$. In this case, the function $r(t)$ satisfies the differential equation

$$
\left(r(t)\left(\lambda^{\prime}(t)\right)\right)^{\prime}=2 r(t) \lambda(t)
$$

for any function $\lambda(t)$ satisfies $\lambda^{\prime}(t) \neq 0$.

Now, we are ready to state and prove the main results when $r(t)>0$. For simplicity, we introduce the following notations:

$$
\left.\begin{array}{ll}
\Phi_{1}\left(Q, r, P_{1,0}\right):=\frac{1}{2 \sqrt{2}}\left[\int_{\alpha}^{\beta} \frac{Q^{2}(t)}{r(t)} P_{1,0}(t) d t\right]^{\frac{1}{2}}, & P_{1,0}(t):=\int_{\alpha}^{t} \frac{(t-s)^{4}}{r(s)} d s, \\
\Phi_{2}\left(Q, r, P_{2,0}\right):=\frac{1}{2 \sqrt{2}}\left[\int_{\alpha}^{\beta} \frac{Q^{2}(t)}{r(t)} P_{2,0}(t) d t\right]^{\frac{1}{2}}, & P_{2,0}(t):=\int_{t}^{\beta} \frac{(s-t)^{4}}{r(s)} d s,
\end{array}\right\}
$$

and

$$
\left.\begin{array}{ll}
\Psi_{1}\left(Q, r, P_{1,1}\right)=\sqrt{\frac{1}{2}}\left[\int_{\alpha}^{\beta} \frac{Q^{2}(t)}{r(t)} P_{1,1}(t)\right]^{\frac{1}{2}}, & P_{1,1}(t)=\int_{\alpha}^{t} \frac{1}{r(s)} d s, \\
\Psi_{2}\left(Q, r, P_{2,1}\right)=\sqrt{\frac{1}{2}}\left[\int_{\alpha}^{\beta} \frac{Q^{2}(t)}{r(t)} P_{2,1}(t)\right]^{\frac{1}{2}}, & P_{2,1}(t)=\int_{t}^{\beta} \frac{1}{r(s)} d s .
\end{array}\right\} .
$$

Theorem 2.2 Suppose that $x$ is a nontrivial solution of $(1.1)$. If $x^{(i)}(\alpha)=0$, for $i=0,1,2$ and $x^{\prime \prime}(\beta)=0$, then

$$
\Phi_{1}\left(Q, r, P_{1,0}\right)+4 \Psi_{1}\left(Q, r, P_{1,1}\right) \geq 1
$$

where $Q(t)=\int_{t}^{\beta} q(s) d s$. If $x^{(i)}(\beta)=0$, for $i=0,1,2$ and $x^{\prime \prime}(\alpha)=0$, then

$$
\Phi_{2}\left(Q, r, P_{2,0}\right)+4 \Psi_{2}\left(Q, r, P_{2,1}\right) \geq 1,
$$

where $Q(t)=\int_{\alpha}^{t} q(s) d s$.

Proof We prove (2.10). Multiplying (1.1) by $x^{\prime \prime}(t)$ and integrating by parts, we have

$$
\begin{aligned}
\int_{\alpha}^{\beta}\left(r(t) x^{\prime \prime \prime}(t)\right)^{\prime} x^{\prime \prime}(t) d t & =\left.r(t) x^{\prime \prime \prime}(t) x^{\prime \prime}(t)\right|_{\alpha} ^{\beta}-\int_{\alpha}^{\beta} r(t)\left(x^{\prime \prime \prime}(t)\right)^{2} d t \\
& =-\int_{\alpha}^{\beta} q(t) x^{\prime \prime}(t) x(t) d t .
\end{aligned}
$$


Using the assumptions $x^{\prime \prime}(\alpha)=x^{\prime \prime}(\beta)=0$, and $Q(t)=\int_{t}^{\beta} q(s) d s$, we have

$$
\int_{\alpha}^{\beta} r(t)\left(x^{\prime \prime \prime}(t)\right)^{2} d t=\int_{\alpha}^{\beta} q(t) x^{\prime \prime}(t) x(t) d t=-\int_{\alpha}^{\beta} Q^{\prime}(t) x^{\prime \prime}(t) x(t) d t .
$$

Integrating by parts the right-hand side, we see that

$$
\begin{aligned}
\int_{\alpha}^{\beta} Q^{\prime}(t) x^{\prime \prime}(t) x(t) d t= & \left.Q(t) x^{\prime \prime}(t) x(t)\right|_{\alpha} ^{\beta}-\int_{\alpha}^{\beta} Q(t) x^{\prime}(t) x^{\prime \prime}(t) d t \\
& -\int_{\alpha}^{\beta} Q(t) x(t) x^{\prime \prime \prime}(t) d t .
\end{aligned}
$$

Using the assumption $x^{\prime \prime}(\beta)=x^{\prime \prime}(\alpha)=0$, we see that

$$
\int_{\alpha}^{\beta} Q^{\prime}(t) x^{\prime \prime}(t) x(t) d t=-\int_{\alpha}^{\beta} Q(t) x^{\prime \prime}(t) x^{\prime}(t) d t-\int_{\alpha}^{\beta} Q(t) x(t) x^{\prime \prime \prime}(t) d t .
$$

Substituting (2.13) into (2.12), we have

$$
\int_{\alpha}^{\beta} r(t)\left|x^{\prime \prime \prime}(t)\right|^{2} d t \leq \int_{\alpha}^{\beta}|Q(t)|\left|x^{\prime}(t)\right|\left|x^{\prime \prime}(t)\right| d t+\int_{\alpha}^{\beta}|Q(t)||x(t)|\left|x^{\prime \prime \prime}(t)\right| d t .
$$

Applying the inequality (2.1) on the integral

$$
\int_{\alpha}^{\beta}|Q(t)||x(t)|\left|x^{\prime \prime \prime}(t)\right| d t
$$

with $\phi(t)=|Q(t)|, \vartheta(t)=r(t), m=1, k=0, l=1, n=3$ and $\mu=2$, we get (note that $x^{(i)}(\alpha)=$ 0 , for $i=0,1,2)$ that

$$
\int_{\alpha}^{\beta}|Q(t)| x(t)\left|x^{\prime \prime \prime}(t)\right| d t \leq \Phi_{1}\left(Q(t), r, P_{1,0}\right)\left[\int_{\alpha}^{\beta} r(t)\left|x^{\prime \prime \prime}(t)\right|^{2} d t\right],
$$

where $\Phi_{1}\left(Q, r, P_{1,0}\right)$ is defined as in (2.8). Applying the inequality (2.1) again on the integral

$$
\int_{\alpha}^{\beta}|Q(t)|\left|x^{\prime}(t)\right|\left|x^{\prime \prime}(t)\right| d t
$$

with $\phi(t)=Q(t), \vartheta(t)=r(t), k=1, n=2, l=m=1$ and $\mu=2$, we see that

$$
\int_{\alpha}^{\beta}|Q(t)|\left|x^{\prime}(t)\right|\left|x^{\prime \prime}(t)\right| d t \leq \Psi_{1}\left(Q, r, P_{1,1}\right)\left[\int_{\alpha}^{\beta} r(t)\left|x^{\prime \prime}(t)\right|^{2} d t\right],
$$

where $\Psi_{1}\left(Q, r, P_{1,1}\right)$ is defined as in (2.9). Applying the Wirtinger inequality (2.5) on the integral

$$
\int_{\alpha}^{\beta} r(t)\left|x^{\prime \prime}(t)\right|^{2} d t
$$

where $x^{\prime \prime}(\alpha)=0=x^{\prime \prime}(\beta)$, we see that

$$
\int_{\alpha}^{\beta} r(t)\left|x^{\prime \prime}(t)\right|^{2} d t \leq 4 \int_{\alpha}^{\beta} r(t)\left|x^{\prime \prime \prime}(t)\right|^{2} d t,
$$


where $r(t)$ satisfies equation (2.7) for any positive function $\lambda(t)$. Substituting (2.17) into (2.16), we have

$$
\int_{\alpha}^{\beta}|Q(t)|\left|x^{\prime}(t)\right|\left|x^{\prime \prime}(t)\right| d t \leq 4 \Psi_{1}\left(Q, r, P_{1,1}\right) \int_{\alpha}^{\beta} r(t)\left|x^{\prime \prime \prime}(t)\right|^{2} d t .
$$

Substituting (2.15) and (2.18) into (2.14) and canceling the term $\int_{\alpha}^{\beta} r(t)\left|x^{\prime \prime \prime}(t)\right|^{2} d t$, we have

$$
K_{1}^{*}\left(Q, r, P_{1,0}\right)+4 \Psi_{1}\left(Q, r, P_{1,1}\right) \geq 1
$$

which is the desired inequality (2.10). The proof of (2.11) is similar by using integration by parts and the constants $\Phi_{1}\left(Q, r, P_{1,0}\right)$ and $\Psi_{1}\left(Q, r, P_{1,1}\right)$ are replaced by $\Phi_{2}\left(Q, r, P_{2,0}\right)$ and $\Psi_{2}\left(Q, r, P_{2,1}\right)$ which are defined as in (2.8). The proof is complete.

Next, we recall the following inequality in Agarwal and Pang [30].

Theorem 2.3 [30] Let $p(t), r(t)$ be nonnegative measurable functions on $(\alpha, \beta)$ and $0 \leq k \leq$ $n-1(n \geq 1)$ fixed. If $x(t) \in C^{n-1}[\alpha, \beta]$ such that $x^{(i)}(\alpha)=0, k \leq i \leq n-1, x^{(n-1)}$ is absolutely continuous on $(\alpha, \beta)$, then

$$
\int_{\alpha}^{\beta} p(t)\left|x^{(k)}(t)\right|\left|x^{(k+1)}(t)\right| d t \leq C_{\alpha} \int_{\alpha}^{\beta} r(t)\left|x^{(n)}(t)\right|^{2} d t
$$

where

$$
C_{\alpha}:=\frac{1}{2((n-k-1) !)^{2}} \max _{t \in[\alpha, \beta]} p(t) \int_{\alpha}^{\beta} \frac{(\beta-s)^{2(n-k-1)}}{r(s)} d s .
$$

If $x(t) \in C^{n-1}[\alpha, \beta]$ such that $x^{(i)}(\beta)=0, k \leq i \leq n-1, x^{(n-1)}$ is absolutely continuous on $(\alpha, \beta)$ then (2.20) holds with $C_{\alpha}$ is replaced by $C_{\beta}$ where

$$
C_{\beta}:=\frac{1}{2((n-k-1) !)^{2}} \max _{t \in[\alpha, \beta]} p(t) \int_{\alpha}^{\beta} \frac{(s-\alpha)^{2(n-k-1)}}{r(s)} d s .
$$

Suppose that the solution $x(t)$ of (1.1) satisfies $x^{\prime}(\alpha)=x^{\prime \prime}(\alpha)=0$. Applying the inequality (2.20) with $p(t)=Q(t), k=1$ and $n=3$ on the term $\int_{\alpha}^{\beta}|Q(t)|\left|x^{\prime}(t)\right|\left|x^{\prime \prime}(t)\right| d t$, leads to

$$
\int_{\alpha}^{\beta}|Q(t)|\left|x^{\prime}(t)\right|\left|x^{\prime \prime}(t)\right| d t \leq C_{2} \int_{\alpha}^{\beta} r(t)\left|x^{\prime \prime \prime}(t)\right|^{2} d t,
$$

where

$$
C_{2}:=\frac{1}{2} \max _{t \in[\alpha, \beta]}|Q(t)| \int_{\alpha}^{\beta} \frac{(\beta-s)^{2}}{r(s)} d s .
$$

If instead $x^{\prime}(\beta)=x^{\prime \prime}(\beta)=0$, then (2.21) holds where $C_{2}$ is replaced by

$$
C_{2}^{*}:=\frac{1}{2} \max _{t \in[\alpha, \beta]}|Q(t)| \int_{\alpha}^{\beta} \frac{(s-\alpha)^{2}}{r(s)} d s .
$$


Using $C_{2}$ and $C_{2}^{*}$ instead of $\Psi_{1}\left(Q, r, P_{1,1}\right)$ and $\Psi_{2}\left(Q, r, P_{2,1}\right)$ in the proof of Theorem 2.2, we obtain the following result.

Theorem 2.4 Suppose that $x$ is a nontrivial solution of $(1.1)$. If $x^{(i)}(\alpha)=0$, for $i=0,1,2$, and $x^{\prime \prime}(\beta)=0$, then

$$
\Phi_{1}\left(Q, r, P_{0,1}\right)+\frac{1}{2} \max _{t \in[\alpha, \beta]}|Q(t)| \int_{\alpha}^{\beta} \frac{(\beta-s)^{2}}{r(s)} d s \geq 1,
$$

where $Q(t)=\int_{t}^{\beta} q(s) d s$. If $x^{(i)}(\beta)=0$, for $i=0,1,2$ and $x^{\prime \prime}(\alpha)=0$, then

$$
\Phi_{2}\left(Q, r, P_{0,2}\right)+\frac{1}{2} \max _{t \in[\alpha, \beta]}|Q(t)| \int_{\alpha}^{\beta} \frac{(s-\alpha)^{2}}{r(s)} d s \geq 1,
$$

where $Q(t)=\int_{\alpha}^{t} q(s) d s$.

If the function $r(t)$ is nonincreasing on $[\alpha, \beta]$, then we see that

$$
\begin{aligned}
{\left[\int_{\alpha}^{\beta} \frac{(\beta-s)^{2}}{r(s)} d s\right] } & \leq \frac{1}{r(\beta)}\left[\int_{\alpha}^{\beta}(\beta-s)^{2} d s\right] \\
& =\frac{(\beta-\alpha)^{3}}{3 r(\beta)},
\end{aligned}
$$

and

$$
\begin{aligned}
{\left[\int_{\alpha}^{\beta} \frac{(s-\alpha)^{2}}{r(s)} d s\right] } & \leq \frac{1}{r(\beta)}\left[\int_{\alpha}^{\beta}(s-\alpha)^{2} d s\right] \\
& =\frac{(\beta-\alpha)^{3}}{3 r(\beta)} .
\end{aligned}
$$

Substituting these last two inequalities into Theorem 2.4, we have the following result.

Theorem 2.5 Assume that $r(t)$ is a nonincreasing function and $x$ is a nontrivial solution of (1.1). If $x^{(i)}(\alpha)=0$, for $i=0,1,2$ and $x^{\prime \prime}(\beta)=0$, then

$$
\begin{gathered}
\Phi_{1}\left(Q, r, P_{1,0}\right)+\frac{(\beta-\alpha)^{3}}{6 r(\beta)} \max _{t \in[\alpha, \beta]}\left|\int_{t}^{\beta} q(s) d s\right| \geq 1 . \\
\text { If } x^{(i)}(\beta)=0, \text { for } i=0,1,2 \text { and } x^{\prime \prime}(\alpha)=0 \text {, then } \\
\Phi_{2}\left(Q, r, P_{2,0}\right)+\frac{(\beta-\alpha)^{3}}{6 r(\beta)} \max _{t \in[\alpha, \beta]}\left|\int_{\alpha}^{t} q(s) d s\right| \geq 1 .
\end{gathered}
$$

We mention here that if we use the maximum value of $|Q(t)|$, we see that

$$
\int_{\alpha}^{\beta}|Q(t)|\left|x^{\prime}(t)\right|\left|x^{\prime \prime}(t)\right| d t \leq \max _{t \in[\alpha, \beta]}|Q(t)| \int_{\alpha}^{\beta}\left|x^{\prime}(t)\right|\left|x^{\prime \prime}(t)\right| d t .
$$

It is worth mentioning here that the inequality due to Fink [31] can be applied on the term $\int_{\alpha}^{\beta}\left|x^{\prime}(t)\right|\left|x^{\prime \prime}(t)\right| d t$. This in fact will give new results when $r(t)$ is a nonincreasing function. 
We now state this inequality. Let $0 \leq k<r<n(n \geq 2)$ but fixed and let $x(t) \in C^{n-1}[0, \beta]$ such that $x^{(i)}(0)=0, k \leq i \leq n-1, x^{(n-1)}$ is absolutely continuous on $(0, \beta)$ and $\frac{1}{\gamma}+\frac{1}{\mu}=1$. Then

$$
\int_{0}^{\beta}\left|x^{(k)}(t)\right|\left|x^{(r)}(t)\right| d t \leq C(n, k, r, \mu) \beta^{2 n-k-r+1-2 / \mu}\left[\int_{0}^{\beta}\left|x^{(n)}(t)\right|^{\mu} d t\right]^{2 / \mu},
$$

where

$$
C(n, k, r, \mu)=\frac{1}{2((n-k-1) !)^{2}[(n-k-1) \gamma+1]^{2 / \gamma}} .
$$

The details of the application of (2.23) will be left to the interested reader. One can note that the inequality (2.23) has been proved without weighted functions, so it will be interesting to extend this inequality and prove an inequality similar to the inequality (2.23) with weighted functions.

In the following, we apply an inequality due to Boyd [32] and the Schwarz inequality to obtain results similar to Theorem 2.5. The Boyd inequality states that if $y \in C^{1}[a, b]$ with $y(a)=0($ or $y(b)=0)$, then

$$
\int_{a}^{b}|y(t)|^{v}\left|y^{\prime}(t)\right|^{\eta} d t \leq N(v, \eta, s)(b-a)^{v}\left(\int_{a}^{b}\left|y^{\prime}(t)\right|^{s} d t\right)^{\frac{v+\eta}{s}},
$$

where $v>0, s>1,0 \leq \eta<s$,

$$
N(v, \eta, s):=\frac{(s-\eta) v^{\nu}}{(s-1)(v+\eta)(I(v, \eta, s))^{\nu}} \sigma^{\nu+\eta-s}, \quad \sigma:=\left\{\frac{v(s-1)+(s-\eta)}{(s-1)(v+\eta)}\right\}^{\frac{1}{s}},
$$

and

$$
I(v, \eta, s):=\int_{0}^{1}\left\{1+\frac{s(\eta-1)}{s-\eta} t\right\}^{-(v+\eta+s v) / s v}[1+(\eta-1) t] t^{1 / v-1} d t .
$$

Note that the inequality (2.24) has immediate application to the case where $y(a)=y(b)=0$. Choose $c=(a+b) / 2$ and apply (2.24) to $[a, c]$ and $[c, b]$, and then add to obtain

$$
\int_{a}^{b}|y(t)|^{\nu}\left|y^{\prime}(t)\right|^{\eta} d t \leq N(v, \eta, s)\left(\frac{b-a}{2}\right)^{v}\left(\int_{a}^{b}\left|y^{\prime}(t)\right|^{s} d t\right)^{\frac{v+\eta}{s}},
$$

where $N(v, \eta, s)$ is defined as in (2.25). The inequality (2.24) has an immediate application when $\eta=s$, to the case where $y(a)=0$ (or $y(b)=0$ ). In this case, equation (2.24) becomes

$$
\int_{a}^{b}|y(t)|^{\nu}\left|y^{\prime}(t)\right|^{\eta} d t \leq L(v, \eta)(b-a)^{\nu}\left(\int_{a}^{b}\left|y^{\prime}(t)\right|^{\eta} d t\right)^{\frac{v+\eta}{\eta}},
$$

where

$$
L(v, \eta):=\frac{\eta v^{\eta}}{v+\eta}\left(\frac{v}{v+\eta}\right)^{\frac{v}{\eta}}\left(\frac{\Gamma\left(\frac{\eta+1}{\eta}+\frac{1}{v}\right)}{\Gamma\left(\frac{\eta+1}{\eta}\right) \Gamma\left(\frac{1}{v}\right)}\right)^{v},
$$

and $\Gamma$ is the Gamma function. 
Saker et al. Journal of Inequalities and Applications 2012, 2012:182

Page 10 of 19

http://www.journalofinequalitiesandapplications.com/content/2012/1/182

Applying the Schwarz inequality,

$$
\int_{\alpha}^{\beta}|f(t) g(t)| d t \leq\left(\int_{\alpha}^{\beta}|f(t)|^{2} d t\right)^{\frac{1}{2}} \times\left(\int_{\alpha}^{\beta}|g(t)|^{2} d t\right)^{\frac{1}{2}}
$$

on the term

$$
\int_{\alpha}^{\beta}|Q(t)|\left|x^{\prime}(t)\right|\left|x^{\prime \prime}(t)\right| d t
$$

we see that

$$
\int_{\alpha}^{\beta}|Q(t)|\left|x^{\prime}(t)\right|\left|x^{\prime \prime}(t)\right| d t \leq\left(\int_{\alpha}^{\beta}|Q(t)|^{2} d t\right)^{\frac{1}{2}}\left(\int_{\alpha}^{\beta}\left|x^{\prime}(t)\right|^{2}\left|x^{\prime \prime}(t)\right|^{2} d t\right)^{\frac{1}{2}} .
$$

Applying the inequality (2.27) on the integral

$$
\int_{\alpha}^{\beta}\left|x^{\prime}(t)\right|^{2}\left|x^{\prime \prime}(t)\right|^{2} d t
$$

with $v=\eta=2$ and $y=x^{\prime}$ (note that $x^{\prime}(\alpha)=0$ ), we see that

$$
\int_{\alpha}^{\beta}\left|x^{\prime}(t)\right|^{2}\left|x^{\prime \prime}(t)\right|^{2} d t \leq\left(\frac{2}{\pi}\right)^{2} \frac{(\beta-\alpha)^{2}}{r^{2}(\beta)}\left[\int_{\alpha}^{\beta} r(t)\left|x^{\prime \prime}(t)\right|^{2} d t\right]^{2}
$$

where we assumed that $r(t)$ is a nonincreasing function. Substituting (2.31) into (2.30), we have

$$
\int_{\alpha}^{\beta}|Q(t)|\left|x^{\prime}(t)\right|\left|x^{\prime \prime}(t)\right| d t \leq \frac{2}{\pi} \frac{(\beta-\alpha)}{r(\beta)}\left(\int_{\alpha}^{\beta}|Q(t)|^{2} d t\right)^{\frac{1}{2}} \int_{\alpha}^{\beta} r(t)\left|x^{\prime \prime}(t)\right|^{2} d t .
$$

Applying the Wirtinger inequality (2.5) on the integral

$$
\int_{\alpha}^{\beta} r(t)\left|x^{\prime \prime}(t)\right|^{2} d t
$$

where $x^{\prime \prime}(\alpha)=0=x^{\prime \prime}(\beta)$, we see that

$$
\int_{\alpha}^{\beta} r(t)\left|x^{\prime \prime}(t)\right|^{2} d t \leq 4 \int_{\alpha}^{\beta} r(t)\left|x^{\prime \prime \prime}(t)\right|^{2} d t
$$

where $r(t)$ satisfies equation (2.7) for any positive function $\lambda(t)$. This implies that

$$
\int_{\alpha}^{\beta}|Q(t)|\left|x^{\prime}(t)\right|\left|x^{\prime \prime}(t)\right| d t \leq 4 \frac{2}{\pi} \frac{(\beta-\alpha)}{r(\beta)}\left(\int_{\alpha}^{\beta}|Q(t)|^{2} d t\right)^{\frac{1}{2}} \int_{\alpha}^{\beta} r(t)\left|x^{\prime \prime \prime}(t)\right|^{2} d t,
$$

where $Q(t)=\int_{t}^{\beta} q(s) d s$. If we replace $x^{\prime}(\alpha)=0$ by $x^{\prime}(\beta)=0$, then (2.32) becomes

$$
\int_{\alpha}^{\beta}|Q(t)|\left|x^{\prime}(t)\right|\left|x^{\prime \prime}(t)\right| d t \leq 4 \frac{2}{\pi} \frac{(\beta-\alpha)}{r(\beta)}\left(\int_{\alpha}^{\beta}|Q(t)|^{2} d t\right)^{\frac{1}{2}} \int_{\alpha}^{\beta} r(t)\left|x^{\prime \prime \prime}(t)\right|^{2} d t,
$$

where $Q(t)=\int_{\alpha}^{t} q(s) d s$. 
Using the inequalities (2.31) and (2.33) and proceeding as in the proof of Theorem 2.4, we obtain the following result.

Theorem 2.6 Assume that $r(t)$ is a nonincreasing function and $x$ is a nontrivial solution of (1.1). If $x^{(i)}(\alpha)=0$, for $i=0,1,2$ and $x^{\prime \prime}(\beta)=0$, then

$$
\begin{aligned}
& \Phi_{1}\left(Q, r, P_{1,0}\right)+4 \frac{2}{\pi} \frac{(\beta-\alpha)}{r(\beta)}\left(\int_{\alpha}^{\beta}\left|\int_{t}^{\beta} q(s) d s\right|^{2} d t\right)^{\frac{1}{2}} \geq 1 . \\
& \text { If } x^{(i)}(\beta)=0 \text {, for } i=0,1,2 \text { and } x^{\prime \prime}(\alpha)=0 \text {, then } \\
& \Phi_{2}\left(Q, r, P_{2,0}\right)+4 \frac{2}{\pi} \frac{(\beta-\alpha)}{r(\beta)}\left(\int_{\alpha}^{\beta}\left|\int_{\alpha}^{t} q(s) d s\right|^{2} d t\right)^{\frac{1}{2}} \geq 1 .
\end{aligned}
$$

Remark 3 In Theorem 2.6, if $x^{\prime}(\alpha)=x^{\prime}(\beta)$, then the term $4 \frac{2}{\pi} \frac{(\beta-\alpha)}{r(\beta)}$ changes to $4 \frac{1}{2 \pi} \frac{(\beta-\alpha)}{r(\beta)}$.

In the following, we will prove some results related to the boundary value problems in the theory of bending of beams. We begin with the boundary conditions $x(\alpha)=x^{\prime}(\alpha)=$ $x(\beta)=x^{\prime}(\beta)=0$, which correspond to a beam clamped at each end. Let

$$
\Psi\left(\left|r^{\prime}\right|, r, P_{1}\right):=\sqrt{\frac{1}{2}}\left[\int_{\alpha}^{\beta} \frac{\left|r^{\prime}(t)\right|^{2}}{r(t)} P_{1}(t)\right]^{\frac{1}{2}}, \quad P_{1}(t):=\int_{\alpha}^{t} \frac{1}{r(s)} d s .
$$

Theorem 2.7 Suppose that $x$ is a nontrivial solution of $(1.1)$ and $Q^{\prime}(t)=q(t)$. If $x(\alpha)=$ $x^{\prime}(\alpha)=x(\beta)=x^{\prime}(\beta)=0$, then

$$
\Psi\left(\left|r^{\prime}\right|, r, P_{1}\right)+\max _{t \in[\alpha, \beta]}|Q(t)| \int_{\alpha}^{\beta} \frac{(\beta-s)^{2}}{r(s)} d s \geq 1,
$$

where $\Psi\left(\left|r^{\prime}(t)\right|, r, P_{1}\right)$ and $P_{1}$ are defined as in (2.34).

Proof Multiplying (1.1) by $x(t)$ and integrating by parts, we have

$$
\begin{aligned}
\int_{\alpha}^{\beta}\left(r(t) x^{\prime \prime \prime}(t)\right)^{\prime} x(t) d t & =\left.r(t) x(t) x^{\prime \prime \prime}(t)\right|_{\alpha} ^{\beta}-\int_{\alpha}^{\beta} r(t) x^{\prime}(t) x^{\prime \prime \prime}(t) d t \\
& =-\int_{\alpha}^{\beta} q(t) x^{2} d t .
\end{aligned}
$$

Using the assumptions that $x(\alpha)=x(\beta)=0$ and $Q^{\prime}(t)=q(t)$, we get that

$$
\int_{\alpha}^{\beta} r(t) x^{\prime}(t) x^{\prime \prime \prime}(t) d t=\int_{\alpha}^{\beta} Q^{\prime}(t) x^{2}(t) d t .
$$

Integrating by parts the right-hand side, we see that

$$
\int_{\alpha}^{\beta} Q^{\prime}(t) x^{2}(t) d t=\left.Q(t) x^{2}(t)\right|_{\alpha} ^{\beta}-2 \int_{\alpha}^{\beta} Q(t) x(t) x^{\prime}(t) d t .
$$


Using the assumption $x(\alpha)=x(\beta)=0$, we see that

$$
\int_{\alpha}^{\beta} Q^{\prime}(t) x^{2}(t) d t=-2 \int_{\alpha}^{\beta} Q(t) x(t) x^{\prime}(t) d t
$$

Integrating by parts the left-hand side of (2.37), we see that

$$
\begin{aligned}
\int_{\alpha}^{\beta} r(t) x^{\prime}(t) x^{\prime \prime \prime}(t) d t= & \left.r(t) x^{\prime}(t) x^{\prime \prime}(t)\right|_{\alpha} ^{\beta}-\int_{\alpha}^{\beta} r^{\prime}(t) x^{\prime}(t) x^{\prime \prime}(t) d t \\
& -\int_{\alpha}^{\beta} r(t)\left(x^{\prime \prime}(t)\right)^{2} d t .
\end{aligned}
$$

Using the assumption $x^{\prime}(\alpha)=x^{\prime}(\beta)=0$, we have

$$
\int_{\alpha}^{\beta} r(t) x^{\prime}(t) x^{\prime \prime \prime}(t) d t=-\int_{\alpha}^{\beta} r^{\prime}(t) x^{\prime}(t) x^{\prime \prime}(t) d t-\int_{\alpha}^{\beta} r(t)\left(x^{\prime \prime}(t)\right)^{2} d t .
$$

Substituting (2.38) and (2.40) into (2.37), we have

$$
\int_{\alpha}^{\beta} r(t)\left|x^{\prime \prime}(t)\right|^{2} d t \leq 2 \int_{\alpha}^{\beta}|Q(t)||x(t)|\left|x^{\prime}(t)\right| d t+\int_{\alpha}^{\beta}\left|r^{\prime}(t)\right|\left|x^{\prime}(t)\right|\left|x^{\prime \prime}(t)\right| d t .
$$

Applying the inequality (2.1) on the integral

$$
\int_{\alpha}^{\beta}\left|r^{\prime}(t)\right|\left|x^{\prime}(t)\right|\left|x^{\prime \prime}(t)\right| d t
$$

with $\phi(t)=\left|r^{\prime}(t)\right|, \vartheta(t)=r(t), k=1, n=2, l=m=1$ and $\mu=2$, we see that

$$
\int_{\alpha}^{\beta}\left|r^{\prime}(t)\right|\left|x^{\prime}(t)\right|\left|x^{\prime \prime}(t)\right| d t \leq \Psi_{1}\left(\left|r^{\prime}(t)\right|, r, P_{1}\right)\left[\int_{\alpha}^{\beta} r(t)\left|x^{\prime \prime}(t)\right|^{2} d t\right],
$$

where $\Psi\left(\left|r^{\prime}(t)\right|, r, P_{1}\right)$ and $P_{1}$ are defined as in (2.34). Applying the inequality (2.20) on the integral

$$
\int_{\alpha}^{\beta}|Q(t)||x(t)|\left|x^{\prime}(t)\right| d t
$$

with $p(t)=Q(t), k=0$ and $n=2$, we see that

$$
\int_{\alpha}^{\beta}|Q(t)||x(t)|\left|x^{\prime}(t)\right| d t \leq \frac{1}{2} \max _{t \in[\alpha, \beta]}|Q(t)| \int_{\alpha}^{\beta} \frac{(\beta-s)^{2}}{r(s)} d s\left[\int_{\alpha}^{\beta} r(t)\left|x^{\prime \prime}(t)\right|^{2} d t\right]
$$

where $x(\alpha)=x^{\prime}(\alpha)=0$. Substituting (2.42) and (2.43) into (2.41) and canceling the term $\left[\int_{\alpha}^{\beta} r(t)\left|x^{\prime \prime}(t)\right|^{2} d t\right]$, we have

$$
\Psi_{1}\left(\left|r^{\prime}(t)\right|, r, P_{1}\right)+\max _{t \in[\alpha, \beta]}|Q(t)| \int_{\alpha}^{\beta} \frac{(\beta-s)^{2}}{r(s)} d s \geq 1,
$$

which is the desired inequality (2.35). The proof is complete. 
Remark 4 One can use the condition $x(\beta)=x^{\prime}(\beta)=0$ instead of $x(\alpha)=x^{\prime}(\alpha)=0$ in the proof of Theorem 2.8. In this case the term $P_{1}(t)=\int_{\alpha}^{t} \frac{1}{r(s)} d s$ is replaced by $P_{2}(t)=\int_{t}^{\beta} \frac{1}{r(s)} d s$ and also the term $\int_{\alpha}^{\beta} \frac{(\beta-s)^{2}}{r(s)} d s$ is replaced by $\int_{\alpha}^{\beta} \frac{(s-\alpha)^{2}}{r(s)} d s$.

In the following, we consider the boundary conditions $x(\alpha)=x^{\prime}(\alpha)=x^{\prime \prime}(\beta)=x^{\prime \prime \prime}(\beta)=0$, which correspond to a beam hinged or supported at both ends. The proof will be as in the proof of Theorem 2.7, by using these boundary conditions to get that $\left.r(t) x^{\prime \prime \prime}(t) x(t)\right|_{\alpha} ^{\beta}=0$ and $\left.r(t) x^{\prime}(t) x^{\prime \prime}(t)\right|_{\alpha} ^{\beta}=0$. This gives us the following result.

Theorem 2.8 Suppose that $x$ is a nontrivial solution of $(1.1)$ and $Q^{\prime}(t)=q(t)$. If $x(\alpha)=$ $x^{\prime}(\alpha)=x^{\prime \prime}(\beta)=x^{\prime \prime \prime}(\beta)=0$, then

$$
\Psi\left(\left|r^{\prime}\right|, r, P_{1}\right)+\max _{t \in[\alpha, \beta]}|Q(t)| \int_{\alpha}^{\beta} \frac{(\beta-s)^{2}}{r(s)} d s \geq 1,
$$

where $\Psi\left(\left|r^{\prime}(t)\right|, r, P_{1}\right)$ and $P_{1}$ are defined as in (2.34).

Next, in the following, we establish some results, which allow us to consider the case when $r(t)<0$. Let

$$
\left.\begin{array}{l}
K_{1}^{*}\left(\left|r(t) r^{\prime}(t)\right|, r^{2}, P_{1,2}\right):=\frac{1}{\sqrt{2}}\left[\int_{\alpha}^{\beta} \frac{\left|r(t) r^{\prime}(t)\right|^{2}}{r^{2}(t)} P_{1,2}(t) d t\right]^{\frac{1}{2}}, \\
K_{2}^{*}\left(\left|r(t) r^{\prime}(t)\right|, r^{2}, P_{2,2}\right):=\frac{1}{\sqrt{2}}\left[\int_{\alpha}^{\beta} \frac{\left|r(t) r^{\prime}(t)\right|^{2}}{r^{2}(t)} P_{2,2}(t) d t\right]^{\frac{1}{2}},
\end{array}\right\}
$$

where

$$
\begin{array}{ll}
P_{1,1}(t):=\int_{\alpha}^{t} \frac{1}{r(s)} d s, & P_{1,2}(t):=\int_{\alpha}^{t} \frac{1}{r(s)} d s, \\
P_{2,1}(t):=\int_{t}^{\beta} \frac{1}{r(s)} d s, & P_{2,2}(t):=\int_{t}^{\beta} \frac{1}{r(s)} d s .
\end{array}
$$

Theorem 2.9 Suppose that $x$ is a nontrivial solution of $(1.1)$. If $x^{(i)}(\alpha)=0$, for $i=0,1,2$ and $x^{\prime \prime}(\beta)=0$, then

$$
\Phi_{1}\left(Q_{1}, r^{2}, P_{1,0}\right)+4 \Psi_{1}\left(Q_{1}, r^{2}, P_{1,1}\right)+K_{1}^{*}\left(\left|r(t) r^{\prime}(t)\right|, r^{2}, P_{1,2}\right) \geq 1,
$$

where $Q_{1}(t)=\int_{t}^{\beta} r(s) q(s) d s$. If $x^{(i)}(\beta)=0$, for $i=0,1,2$ and $x^{\prime \prime}(\alpha)=0$, then

$$
\Phi_{2}\left(Q_{1}, r^{2}, P_{2,0}\right)+4 \Psi_{2}\left(Q_{1}, r^{2}, P_{2,1}\right)+K_{2}^{*}\left(\left|r(t) r^{\prime}(t)\right|, r^{2}, P_{2,2}\right) \geq 1,
$$

where $Q_{1}(t)=\int_{\alpha}^{t} r(s) q(s) d s$.

Proof We prove (2.45). Multiplying (1.1) by $r x^{\prime \prime}(t)$ and integrating by parts, we have

$$
\begin{aligned}
\int_{\alpha}^{\beta}\left(r(t) x^{\prime \prime \prime}(t)\right)^{\prime} r(t) x^{\prime \prime}(t) d t= & \left.r^{2}(t) x^{\prime \prime \prime}(t) x^{\prime \prime}(t)\right|_{\alpha} ^{\beta}-\int_{\alpha}^{\beta} r^{2}(t)\left(x^{\prime \prime \prime}(t)\right)^{2} d t \\
& -\int_{\alpha}^{\beta} r(t) r^{\prime}(t) x^{\prime \prime}(t) x^{\prime \prime \prime}(t) d t \\
= & -\int_{\alpha}^{\beta} r(t) q(t) x^{\prime \prime}(t) x(t) d t .
\end{aligned}
$$


Using the assumptions $x^{\prime \prime}(\alpha)=x^{\prime \prime}(\beta)=0$ and $Q_{1}(t)=\int_{t}^{\beta} r(s) q(s) d s$, we have

$$
\begin{aligned}
\int_{\alpha}^{\beta} r^{2}(t)\left|x^{\prime \prime \prime}(t)\right|^{2} d t & =-\int_{\alpha}^{\beta} r(t) r^{\prime}(t) x^{\prime \prime}(t) x^{\prime \prime \prime}(t) d t+\int_{\alpha}^{\beta} q(t) x(t) x^{\prime \prime}(t) d t \\
& =-\int_{\alpha}^{\beta} r(t) r^{\prime}(t) x^{\prime \prime}(t) x^{\prime \prime \prime}(t) d t-\int_{\alpha}^{\beta} Q_{1}^{\prime}(t) x(t) x^{\prime \prime}(t) d t .
\end{aligned}
$$

Integrating by parts the last term in the right-hand side, we see that

$$
\begin{aligned}
\int_{\alpha}^{\beta} Q_{1}^{\prime}(t) x^{\prime \prime}(t) x(t) d t= & \left.Q_{1}(t) x^{\prime \prime}(t) x(t)\right|_{\alpha} ^{\beta}-\int_{\alpha}^{\beta} Q_{1}(t) x^{\prime}(t) x^{\prime \prime}(t) d t \\
& -\int_{\alpha}^{\beta} Q_{1}(t) x(t) x^{\prime \prime \prime}(t) d t .
\end{aligned}
$$

Using the assumption $x^{\prime \prime}(\beta)=x^{\prime \prime}(\alpha)=0$, we see that

$$
\int_{\alpha}^{\beta} Q^{\prime}(t) x(t) x^{\prime \prime}(t) d t=-\int_{\alpha}^{\beta} Q_{1}(t) x^{\prime}(t) x^{\prime \prime}(t) d t-\int_{\alpha}^{\beta} Q_{1}(t) x(t) x^{\prime \prime \prime}(t) d t
$$

Substituting (2.13) into (2.12), we have

$$
\begin{aligned}
\int_{\alpha}^{\beta} r^{2}(t)\left|x^{\prime \prime \prime}(t)\right|^{2} d t \leq & \int_{\alpha}^{\beta}\left|Q_{1}(t)\right||x(t)|\left|x^{\prime \prime \prime}(t)\right| d t+\int_{\alpha}^{\beta}\left|Q_{1}(t)\right|\left|x^{\prime}(t)\right|\left|x^{\prime \prime}(t)\right| d t \\
& +\int_{\alpha}^{\beta}\left|r(t) r^{\prime}(t)\right|\left|x^{\prime \prime}(t)\right|\left|x^{\prime \prime \prime}(t)\right| d t .
\end{aligned}
$$

Applying the inequality (2.1) on the integral

$$
\int_{\alpha}^{\beta}\left|Q_{1}(t)\right||x(t)|\left|x^{\prime \prime \prime}(t)\right| d t
$$

with $\phi(t)=\left|Q_{1}(t)\right|, \vartheta(t)=r(t), m=1, k=0, l=1, n=3$, and $\mu=2$, we get (note that $x^{(i)}(\alpha)=$ 0 , for $i=0,1,2)$ that

$$
\int_{\alpha}^{\beta}\left|Q_{1}(t)\right| x(t)\left|x^{\prime \prime \prime}(t)\right| d t \leq \Phi_{1}\left(\left|Q_{1}(t)\right|, r^{2}, P_{1,0}\right)\left[\int_{\alpha}^{\beta} r(t)\left|x^{\prime \prime \prime}(t)\right|^{2} d t\right]
$$

where $\Phi_{1}\left(Q_{1}, r, P_{1,0}\right)$ is defined as in (2.8) and $Q$ is replaced by $Q_{1}$. Applying the inequality (2.1) again on the integral

$$
\int_{\alpha}^{\beta}\left|Q_{1}(t)\right|\left|x^{\prime}(t)\right|\left|x^{\prime \prime}(t)\right| d t
$$

with $\phi(t)=Q_{1}(t), \vartheta(t)=r(t), k=1, n=2, l=m=1$ and $\mu=2$, we see that

$$
\int_{\alpha}^{\beta}\left|Q_{1}(t)\right|\left|x^{\prime}(t)\right|\left|x^{\prime \prime}(t)\right| d t \leq \Psi_{1}\left(Q_{1}, r^{2}, P_{1,1}\right)\left[\int_{\alpha}^{\beta} r^{2}(t)\left|x^{\prime \prime}(t)\right|^{2} d t\right],
$$


where $\Psi_{1}\left(Q_{1}, r, P_{1,1}\right)$ is defined as in (2.9) and $Q$ is replaced by $Q_{1}$. Applying the Wirtinger inequality (2.5) on the integral

$$
\int_{\alpha}^{\beta} r^{2}(t)\left|x^{\prime \prime}(t)\right|^{2} d t
$$

where $x^{\prime \prime}(\alpha)=0=x^{\prime \prime}(\beta)$, we see that

$$
\int_{\alpha}^{\beta} r^{2}(t)\left|x^{\prime \prime}(t)\right|^{2} d t \leq 4 \int_{\alpha}^{\beta} r^{2}(t)\left|x^{\prime \prime \prime}(t)\right|^{2} d t
$$

where $r^{2}(t)$ satisfies equation (2.7) for any positive function $\lambda(t)$. Substituting (2.52) into (2.51), we have

$$
\int_{\alpha}^{\beta}\left|Q_{1}(t)\right|\left|x^{\prime}(t)\right|\left|x^{\prime \prime}(t)\right| d t \leq 4 \Psi_{1}\left(Q_{1}, r^{2}, P_{1,1}\right) \int_{\alpha}^{\beta} r(t)\left|x^{\prime \prime \prime}(t)\right|^{2} d t .
$$

Applying the inequality (2.1) on the integral

$$
\int_{\alpha}^{\beta}\left|r(t) r^{\prime}(t)\right|\left|x^{\prime \prime}(t)\right|\left|x^{\prime \prime \prime}(t)\right| d t
$$

with $\phi(t)=\left|r(t) r^{\prime}(t)\right|, \vartheta(t)=r(t), m=1, k=2, l=1, n=3$, and $\mu=2$, we get (note that $x^{(i)}(\alpha)=0$, for $\left.i=0,1,2\right)$ that

$$
\int_{\alpha}^{\beta}\left|r(t) r^{\prime}(t)\right|\left|x^{\prime \prime}(t)\right|\left|x^{\prime \prime \prime}(t)\right| d t \leq K_{1}^{*}\left(\left|r(t) r^{\prime}(t)\right|, r^{2}, P_{1,2}\right)\left[\int_{\alpha}^{\beta} r^{2}(t)\left|x^{\prime \prime \prime}(t)\right|^{2} d t\right],
$$

where $K_{1}^{*}\left(Q, r^{2}, P_{1,2}\right)$ is defined as in (2.44). Substituting (2.50), (2.53), and (2.54) into (2.49) and canceling the term $\int_{\alpha}^{\beta} r^{2}(t)\left|x^{\prime \prime \prime}(t)\right|^{2} d t$, we have

$$
\Phi_{1}\left(Q_{1}, r^{2}, P_{1,0}\right)+4 \Psi_{1}\left(Q_{1}, r^{2}, P_{1,1}\right)+K_{1}^{*}\left(\left|r(t) r^{\prime}(t)\right|, r^{2}, P_{1,2}\right) \geq 1,
$$

which is the desired inequality (2.45). The proof of (2.46) is similar to (2.45) by using the integration by parts and the constants

$$
\Phi_{1}\left(Q_{1}, r^{2}, P_{1,0}\right), \quad \Psi_{1}\left(Q_{1}, r^{2}, P_{1,1}\right), \quad K_{1}^{*}\left(\left|r(t) r^{\prime}(t)\right|, r^{2}, P_{1,2}\right),
$$

are replaced by

$$
\Phi_{2}\left(Q_{1}, r^{2}, P_{2,0}\right), \quad \Psi_{2}\left(Q_{1}, r^{2}, P_{2,1}\right), \quad K_{2}^{*}\left(\left|r(t) r^{\prime}(t)\right|, r^{2}, P_{2,2}\right),
$$

which are defined as in (2.8) and (2.44). The proof is complete.

\section{Discussions and examples}

In this section, we present some special cases of the results obtained in Section 2 and also give some illustrative examples. We begin with Theorem 2.2 and consider the case when $r(t)=1$. In this case, equation (1.1) becomes the fourth-order differential equation

$$
x^{(4)}(t)+q(t) x(t)=0, \quad t \in[\alpha, \beta] .
$$


Using the definitions of the functions $P_{1,0}$ and $P_{2,0}$, and putting $r(t)=1$, we see after simplifications that

$$
P_{1,0}(t):=\int_{\alpha}^{t}(t-s)^{4} d s=\frac{(t-\alpha)^{5}}{5}, \quad P_{2,0}(t):=\int_{t}^{\beta}(s-t)^{4} d s=\frac{(\beta-t)^{5}}{5} .
$$

which lead to

$$
\begin{aligned}
& \Phi_{1}\left(Q, r, P_{1,0}\right) \leq \frac{1}{4 \sqrt{15}} \max _{t \in[\alpha, \beta]}|Q(t)|(\beta-\alpha)^{3}, \\
& \Phi_{2}\left(Q, r, P_{2,0}\right) \leq \frac{1}{4 \sqrt{15}} \max _{t \in[\alpha, \beta]}|Q(t)|(\beta-\alpha)^{3},
\end{aligned}
$$

and

$$
\begin{aligned}
& \Psi_{1}\left(Q, r, P_{1,1}\right) \leq \frac{1}{2} \max _{t \in[\alpha, \beta]}|Q(t)|(\beta-\alpha), \\
& \Psi_{2}\left(Q, r, P_{2,1}\right) \leq \frac{1}{2} \max _{t \in[\alpha, \beta]}|Q(t)|(\beta-\alpha) .
\end{aligned}
$$

As a special case of Theorem 2.2, if $r(t)=1$, we have the following result.

Theorem 3.1 Suppose that $x$ is a nontrivial solution of $(3.1)$. If $x^{(i)}(\alpha)=0$, for $i=0,1,2$ and $x^{\prime \prime}(\beta)=0$, then

$$
\frac{1}{4 \sqrt{15}} \max _{t \in[\alpha, \beta]}|Q(t)|\left[(\beta-\alpha)^{3}+8 \sqrt{15}(\beta-\alpha)\right] \geq 1
$$

where $Q(t)=\int_{t}^{\beta} q(s) d s$. If $x^{(i)}(\beta)=0$, for $i=0,1,2$ and $x^{\prime \prime}(\alpha)=0$, then

$$
\frac{1}{4 \sqrt{15}} \max _{t \in[\alpha, \beta]}|Q(t)|\left[(\beta-\alpha)^{3}+8 \sqrt{15}(\beta-\alpha)\right] \geq 1
$$

where $Q(t)=\int_{\alpha}^{t} q(s) d s$.

As a special case of Theorem 2.5, if $r(t)=1$, then we have the following result.

Theorem 3.2 Suppose that $x$ is a nontrivial solution of $(3.1)$. If $x^{(i)}(\alpha)=0$, for $i=0,1,2$ and $x^{\prime \prime}(\beta)=0$, then

$$
\max _{t \in[\alpha, \beta]}\left|\int_{t}^{\beta} q(s) d s\right| \geq \frac{4 \sqrt{15}+6}{(\beta-\alpha)^{3}} .
$$

If $x^{(i)}(\beta)=0$, for $i=0,1,2$ and $x^{\prime \prime}(\alpha)=0$, then

$$
\max _{t \in[\alpha, \beta]}\left|\int_{\alpha}^{t} q(s) d s\right| \geq \frac{4 \sqrt{15}+6}{(\beta-\alpha)^{3}} .
$$

As a special case of Theorem 2.7, if $r(t)=1$, we have the following result. 
Theorem 3.3 Suppose that $x$ is a nontrivial solution of $(3.1)$. If $x(\alpha)=x^{\prime}(\alpha)=x(\beta)=x^{\prime}(\beta)=$ 0 , then

$$
\max _{t \in[\alpha, \beta]}\left|\int_{\alpha}^{t} q(t) d t\right| \geq \frac{3}{(\beta-\alpha)^{3}}
$$

Remark 5 Note that the violation of the conditions in Theorem 3.3 yield sufficient conditions for disconjugacy of equation (3.1).

As a special case of Theorem 2.8, if $r(t)=1$, we have the following result.

Theorem 3.4 Suppose that $x$ is a nontrivial solution of (3.1). If $x(\alpha)=x^{\prime}(\alpha)=x^{\prime \prime}(\beta)=$ $x^{\prime \prime \prime}(\beta)=0$, then

$$
\max _{t \in[\alpha, \beta]}\left|\int_{\alpha}^{t} q(t) d t\right| \geq \frac{3}{(\beta-\alpha)^{3}} .
$$

The following examples illustrate the results.

Example 1 Consider the equation

$$
x^{(4)}(t)+\lambda \cos ^{2}(\alpha t) x(t)=0, \quad 0 \leq t \leq \pi,
$$

where $\lambda$ and $\alpha$ are positive constants. If $x(t)$ be a solution of $(3.3)$ with $x(0)=x^{\prime}(0)=x(\pi)=$ $x^{\prime}(\pi)=0$, then

$$
\lambda \max _{t \in[0, \pi]} \int_{0}^{t} \cos ^{2}(\alpha t) d t=\lambda \max _{t \in[0, \pi]}\left[\frac{1}{2} t+\frac{1}{4 \alpha} \sin (2 \alpha t)\right]=\frac{\lambda \pi}{2}+\frac{\lambda}{4 \alpha} .
$$

Then if $\lambda \geq 1$, the condition (3.2) of Theorem 3.3 is given by

$$
\frac{\lambda \pi}{2}+\frac{\lambda}{4 \alpha} \geq \frac{3}{\pi^{3}}
$$

which is satisfied for any $\lambda \geq 1$ and $\alpha>0$.

Example 2 Consider the equation

$$
x^{(4)}(t)+\lambda \cos (\alpha t) x(t)=0, \quad 0 \leq t \leq \beta,
$$

where $\lambda$ and $\alpha$ are positive constants. By Theorem 3.3, we see that the equation (3.5) is 2-2 disconjugate on $[0, \beta]$ if

$$
\max _{t \in[\alpha, \beta]}\left|\int_{\alpha}^{t} q(t) d t\right|=\max _{t \in[0, \beta]} \lambda\left|\int_{0}^{t} \cos (\alpha t) d t\right|:=\frac{\lambda}{\alpha}<\frac{3}{\beta^{3}} .
$$

That is,

$$
\beta<\left(\frac{3}{\lambda}\right)^{\frac{1}{3}} \alpha^{\frac{1}{3}}
$$


From this, we conclude that the interval of disconjugacy is bounded below by a constant times the cubic root of the frequency $\alpha^{\frac{1}{3}}$ for $\alpha \geq 1$, i.e., if $[0, \beta)$ is the interval of disconjugacy, then $\beta>\left(\frac{3}{\lambda}\right)^{\frac{1}{3}} \alpha^{\frac{1}{3}}$. In fact, this is compatible with the special case of the results that has been proved in [33].

\section{Competing interests}

The authors declare that they have no competing interests.

\section{Authors' contributions}

All the authors contributed equaly and significantly in writting this paper.

\section{Author details}

${ }^{1}$ Department of Mathematics, Faculty of Science, Mansoura University, Mansoura, 35516, Egypt. ${ }^{2}$ Department of Mathematics, Texas A and M University-Kingsville, Kingsville, Texas 78363, USA. ${ }^{3}$ School of Mathematics, Statistics and Applied Mathematics, National University of Ireland, Galway, Ireland.

Received: 30 January 2012 Accepted: 1 August 2012 Published: 30 August 2012

\section{References}

1. Lyapunov, AM: Probleme General de la Stabilitie du Movement. Ann. of Math. Stud., vol. 17. Princeton University Press, Princeton (1949)

2. Wintner, A: On the nonexistence of conjugate points. Amer. J. Math. 73, 368-380 (1951)

3. Almenar, P, Jódar, L: A Lyapunov inequality for a second order nonlinear differential equations. Appl. Math. Lett. 24, 524-527 (2001)

4. Cakmak, D: Lyapunov-type integral inequalities for certain higher order differential equations. Appl. Math. Comput. 216, 368-373 (2010)

5. Chen, LS: A Lyapunov inequality and forced oscillations in general nonlinear nth order differential-difference equations. Glasg. Math. J. 18, 161-166 (1977)

6. Eliason, SB: Lyapunov type inequalities for certain second-order functional differential equations. SIAM J. Appl. Math. 27, 180-199 (1974)

7. Parhi, N, Panigrahi, S: On Liapunov-type inequality for third order differential equations. J. Math. Anal. Appl. 233 445-460 (1999)

8. Parhi, N, Panigrahi, S: Disfocality and Liapunov-type inequalities for third-order differential equations. Comput. Math. Appl. 16, 227-233 (2003)

9. Pachpatte, BG: A note on Lyapunov type inequalities. Indian J. Pure Appl. Math. 21, 45-49 (1990)

10. Pachpatte, BG: On the zeros of solutions of certain differential equations. Demonstr. Math. 25, 825-833 (1992)

11. Pachpatte, BG: A Lyapunov type inequality for a certain second-order differential equation. Proc. Natl. Acad. Sci., India 64, 69-73 (1994)

12. Pachpatte, BG: An inequality suggested by Lyapunov's inequality. Centre Rech. Math. Pures, Neuchatel Chambery, Fac. 26, Ser. I, 1-4 (1995)

13. Pachpatte, BG: Lyapunov type integral inequalities for certain differential equations. Georgian Math. J. 4, 139-148 (1997)

14. Pachpatte, BG: Inequalities related to the zeros of solutions of certain second order differential equations. Facta Univ. Ser. Math. Inform. 16, 35-44 (2001)

15. Patula, WT: On the distance between zeros. Proc. Am. Math. Soc. 52, 247-251 (1975)

16. Panigrahi, S: Lyapunov-type integral inequalities for certain higher-order differential equations. Electron. J. Differ. Equ. 2009, 1-14 (2009)

17. Reid, WT: A generalized Lyapunov inequality. Electron. J. Differ. Equ. 13, 182-196 (1973)

18. Saker, SH: Lyapunov inequalities for half-linear dynamic equations on time scales and disconjugacy. Dyn. Contin. Discrete Impuls. Syst., Ser. B, Appl. Algorithms 18, 149-161 (2011)

19. Saker, SH: Applications of Wirtinger inequalities on the distribution of zeros of the Riemann Zeta-function. J. Inequal. Appl. 2010, $215416(2010)$

20. Saker, SH: Applications of Opial and Wirtinger inequalities on zeros of third order differential equations. Dyn. Syst. Appl. 20, 479-494 (2011)

21. Saker, SH: Lyapunov type inequalities for a second order differential equations with a damping term. Ann. Pol. Math. 103, 37-57 (2012)

22. Saker, SH: Opial's type inequalities on time scales and some applications. Ann. Pol. Math. 104, 234-260 (2012)

23. Nehari, Z: Green's functions and disconjugacy. Arch. Ration. Mech. Anal. 62, 53-76 (1976)

24. Elias, U: Necessary conditions and sufficent conditions for disfocality and disconjugacy of a differential equation. Pac. J. Math. 81, 379-397 (1979)

25. Yang, X: On a Liapunov-type inequality for a certain higher-order differential equations. Appl. Math. Comput. 134, 307-317 (2003)

26. Courant, R, Hilbert, D: Methods of Mathematical Physics, vol. I, Wiley, New York (1989)

27. Zill, DG, Wright, WS, Cullen, MR: Advanced Engineering Mathematics, 4th edn., Jones and Bartlett Publishers, Canada (2011)

28. Agarwal, RP, Pang, PYH: Opial Inequalities with Applications in Differential and Difference Equations, Kluwer, Dordrechet (1995) 
29. Agarwal, RP, Bohner, M, O'Regan, D, Saker, SH: Some dynamic Wirtinger-type inequalities and their applications. Pac. J. Math. 252, 1-18 (2011)

30. Agarwal, RP, Pang, PYH: Sharp Opial-type inequalities involving higher order derivatives of two functions. Math. Nachr. 174, 5-20 (1995)

31. Fink, AM: On Opial's inequality for $f^{n}$. Proc. Am. Math. Soc. 115, 177-181 (1992)

32. Boyd, D: Best constants in class of integral inequalities. Pac. J. Math. 30, 367-383 (1969)

33. Brown, R, Hinton, D: Lyapunov inequalities and their applications. In: Rassias, T (ed.) Survey on Classical Inequalities, pp. 1-25. Kluwer, Drodrecht, (2000)

doi:10.1186/1029-242X-2012-182

Cite this article as: Saker et al.: New gaps between zeros of fourth-order differential equations via Opial inequalities. Journal of Inequalities and Applications 2012 2012:182.

Submit your manuscript to a SpringerOpen ${ }^{\circ}$ journal and benefit from:

- Convenient online submission

- Rigorous peer review

- Immediate publication on acceptance

- Open access: articles freely available online

- High visibility within the field

- Retaining the copyright to your article

Submit your next manuscript at $>$ springeropen.com 